\title{
Artificial finger joint replacement due to a giant cell tumor of the tendon sheath with bone destruction: A case report
}

\author{
HUI LU, HUI SHEN, QIANG CHEN, XIANG-QIAN SHEN and SHOU-CHENG WU \\ Department of Hand Surgery, The First Affiliated Hospital, College of Medicine, Zhejiang University, \\ Hangzhou, Zhejiang 310003, P.R. China
}

Received October 24, 2014; Accepted September 25, 2015

DOI: $10.3892 / \mathrm{ol} .2015 .3813$

\begin{abstract}
The current study presents the case of a 25-year-old male who developed tumor recurrence of the proximal phalange of the ring finger on the right hand 4 years after partial tumor resection surgery. An X-ray of the right hand showed that the distal bone of the proximal phalange on the ring finger was destroyed. An artificial finger joint replacement was performed using a silicone joint for this unusual tumor recurrence. The pathological findings were indicative of a giant cell tumor of the tendon sheath. As a result of surgery, the patient's proximal interphalangeal point motion recovered to the pre-operative level. The pre-operative and post-operative disabilities of the arm, at shoulder and hand and total activity measurement values were 1.67 and 3.33 , and 255 and $243^{\circ}$, respectively. Complications such as tumor recurrence, joint dislocation and the requirement for prosthetic training were not observed during the 5-year follow-up period.
\end{abstract}

\section{Introduction}

Giant cell tumor of the tendon sheath (GCTTS) is also known as giant-cell synovioma and localized nodular tenosynovitis. GCTTS is most commonly attached to the tendons of the fingers, hands, and wrists, and affects both males and females between the ages of 20 and 50 (1). It is a common treatment to remove the tumor by the surgery, after which it has a high recurrence (2). GCTTS with bone destruction occurs rarely and is hard to remove completely (3). Previous case studies have shown that traditional surgical methods impact joint function and that the tumors easily recur (3-5). The present study reports the case of a young patient with a GCTTS who underwent an artificial finger joint replacement; the tumor was

Correspondence to: Dr Hui Lu, Department of Hand Surgery, The First Affiliated Hospital, College of Medicine, Zhejiang University, 79 Qingchun Road, Hangzhou, Zhejiang 310003, P.R. China E-mail: hitman1982@hotmail.com

Key words: artificial finger joint, bone destruction, finger joint, giant cell tumor of the tendon sheath, tumor excised completely and the function of the joints was recovered.

\section{Case report}

A 25-year-old male was admitted to The First Affiliated Hospital, College of Meicine, ZheJiang University (Hangzhou, Zhejiang, China) with tumor recurrence of the proximal phalange of the ring finger on the right hand 4 years after partial tumor resection surgery. The patient had no history of trauma or infection and was previously healthy. A physical examination revealed swelling of the proximal phalange of the finger without clear cause, multiple nodules in the local areas of the proximal interphalangeal point (PIP) without marked pain, and a lack of phalangeal paresthesia, a blood circulation disorder or a finger movement disorder. An X-ray of the right hand showed that the distal bone of the proximal phalange of the ring finger on the right hand had been destroyed. Although the diagnosis was unfavorable, a local resection of the extra-articular joint was performed to remove the tumor mass. The surgical pathology report confirmed GCTSS. The tumor recurred after 1 year, with multiple nodules found in the proximal phalange. According to the physical examination, the soft tissue in the PIP of the finger was swollen, but no skin redness was found. Multiple nodules could be locally palpated with mild tenderness. The blood supply to the fingertip was good, and the range of motion of the PIP was satisfactory. An X-ray of the right hand showed distal bone destruction of the proximal phalange of the ring finger on the right hand and swelling of the soft tissue. Magnetic resonance image of the finger (Fig. 1) showed that the distal bone of the proximal phalange of the finger was structurally destroyed, and the adjacent PIP capsule and periarticular soft tissue were markedly swollen. Slightly elongated T1- and T2-weighted signals were abnormal. The joint space remained. A giant cell tumor of the bone was subsequently diagnosed (Figs. 2 and 3).

Resection of the tumor of the distal phalange was performed under general anesthesia. Local swelling was found around the PIP of the ring finger. Following incision of the skin and subcutaneous tissue of the dorsal digit, the bulk of the tumor was observed to be derived from the extensor tendon, with infiltration into the PIP capsule and ligament. The local soft tissue was severely damaged. The tumor mass was yellow-brown in color. Subsequent to removal of the tumor and involved soft 


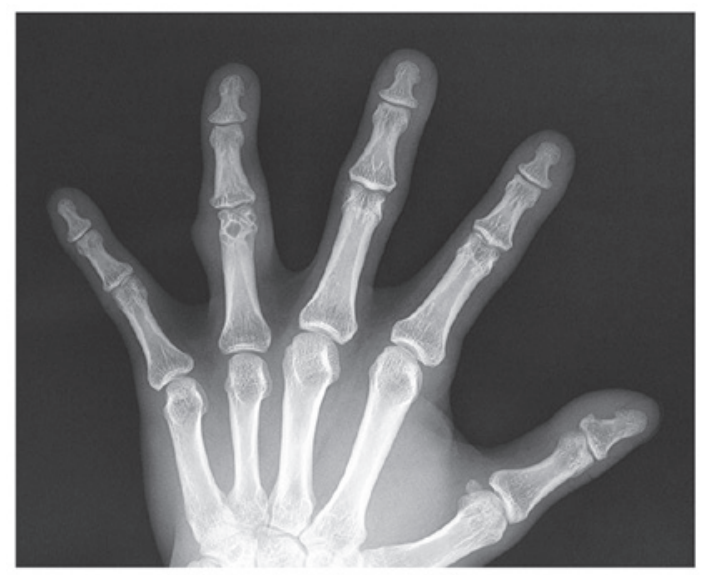

Figure 1. An X-ray of the right hand showing distal bone destruction of the proximal phalange of the ring finger and swelling of the soft tissue.

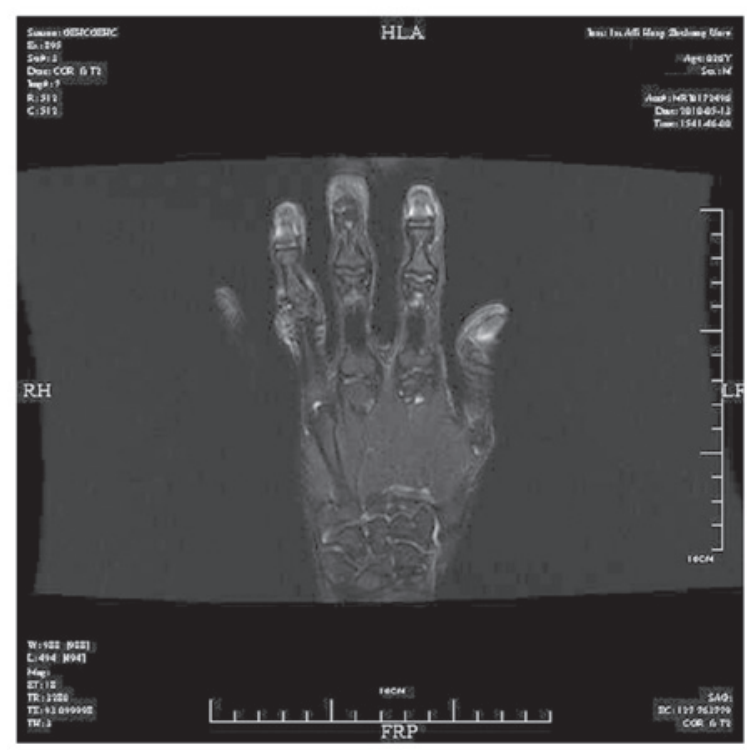

Figure 2. Slightly elongated T1-weighted signal on magnetic resonance imaging.

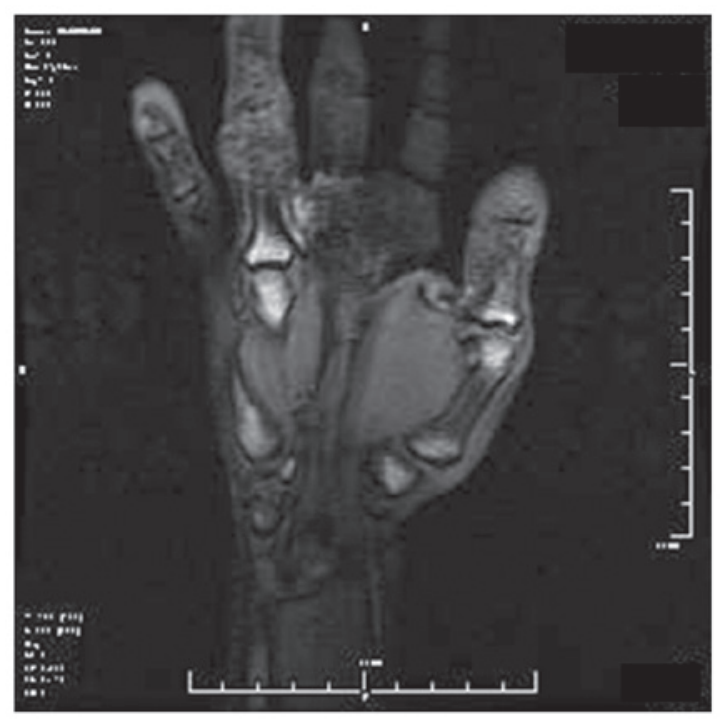

Figure 3. Abnormal T2-weighted signals on magnetic resonance imaging. The joint space remained. A giant cell tumor of the bone was diagnosed.

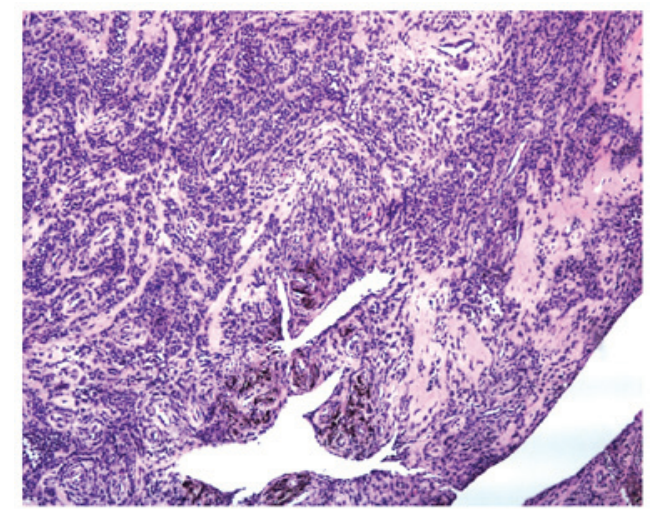

Figure 4. Pathological examination indicative of a giant cell tumor of the tendon sheath. The tumor consists of large rounded synoviocytes and a small number of multinuclear giant cells, arranged in diffuse sheets. Extensive hemosiderin deposition is also visible (stain, hematoxylin and eosin; magnification, $\mathrm{x} 50$ ).

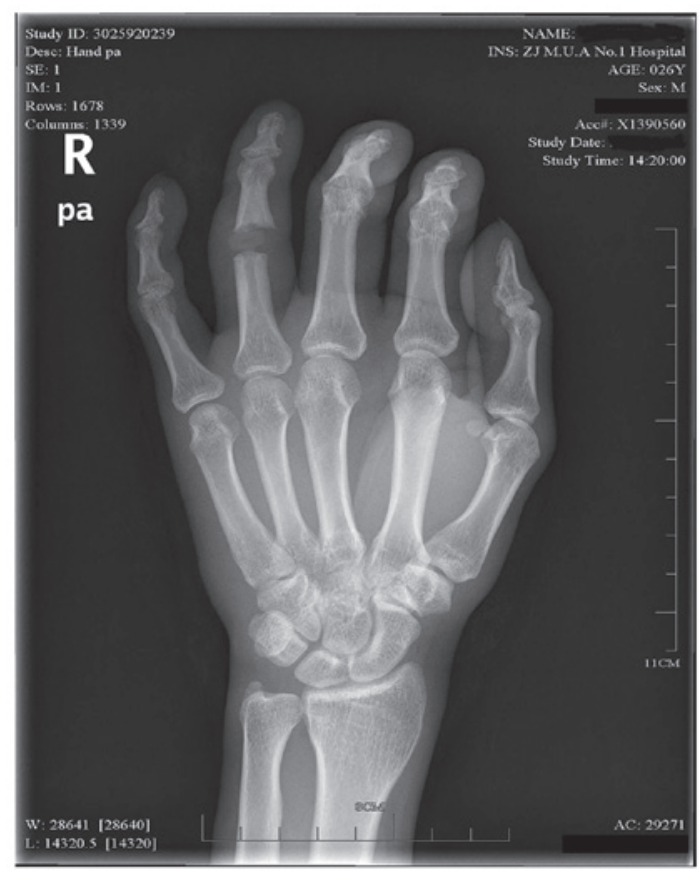

Figure 5. X-ray at 24 months post-surgery.

tissue, and opening of the joint capsule, it was found that the tumor had infiltrated into the joint and destroyed the bone. Part of the tumor reached the joint, causing severe local damage of the bone. No indication of a simple tumor resection was found. Subsequently, an osteotomy was performed in the segment $0.5 \mathrm{~cm}$ from the proximal and distal regions of the joint to completely remove the tumor tissue. No significant tumor infiltration was found in the palmar tendon or soft tissue. A drill was used to expand the space to encompass nearly the entire bone marrow cavity of the intermediate phalange, and a Swanson \#2 artificial silicone joint (Wright Medical Technology, Inc., Memphis, TN, USA) was placed inside the joint space. The residual dorsal capsule and ligaments on either side were sutured to strengthen and protect the joint. The wound was repeatedly washed. The local soft tissue and skin were sutured and wrapped with aseptic dressing. The treated finger 
was fixed in a dorsiflexion position with an external plaster support.

Irregular tissue measuring $2 \times 1.2 \times 0.2 \mathrm{~cm}$ was submitted for examination. Upon magnification, the tumor was found to be composed of a large number of round and synovial cells and a few multinucleated giant cells. The tumor was lamellar in arrangement, and a large amount of hemosiderin deposition was found. These findings were indicative of a GCTTS (Fig. 4).

At 3-5 days post-surgery, the external plaster was removed and functional exercises were begun. Orderly and gradual exercise of the passive joint was conducted early during recovery, with three groups of exercises being performed every day, and each group being performed 10 times, while avoiding joint dislocation and tendon rupture. Once the patient's pain and local inflammatory edema subsided, active motion of the joint was gradually conducted. A quantitative exercise program was begun based on the use of a hand continuous passive motion machine. At discharge, the patient's PIP motion had recovered to the pre-operative level (Fig. 5), with pre-operative and post-operative disabilities of the arm, at shoulder and hand, and total activity measurement values of 1.67 and 3.33, and 255 and $243^{\circ}$, respectively. Complications such as tumor recurrence, joint dislocation and the requirement for prosthetic training were not observed during a 5-year follow-up period.

\section{Discussion}

GCTTS is a common benign tumor arising in the tendon sheath GCTTS occurs most often in the hands and feet (6). Pigmented villonodular synovitis (PVNS) is a rare, idiopathic proliferative disorder of the synovium (7). It usually affects the hip or keen, but also occurs in hand or foot and the joint becomes swollen. Therefore, PVNS is often confused with GCTTS. The use of MRI may show a combination of synovial proliferation and soft tissue masses, together with deposits of hemosiderin and bone erosion, is highly diagnostic for PVNS, may also adequately displays the location and extent of the lesion and its internal structure (8).

Studies on GCTTS with bone destruction are inconsistent. In a report by Moore et al, no bone destruction was found among 115 cases of GCTTS (9). Bone destruction was detected in only 1 out of 51 cases of GCTTS reported by Fyfe and MacFarlane (10). Jones et al (11) studied 91 cases of GCTTS in the hand, among which 11 cases showed evidence of cortical bone erosion without bone destruction. However, certain studies have suggested that GCTTS with bone destruction is not uncommon. According to a study by Pan et al (12), among 98 cases, the bone was normal in 55 cases, the tumor infiltrated into the bone surface in 16 cases and the bone was destroyed in 27 cases. In a study by Uriburu and Levy (13), 23.3\% of 133 total cases showed tumor intruding onto the bone surface and $11.3 \%$ exhibited bone destruction. Additionally, in another study, X-ray analysis showed lucent areas in single locular or multilocular cysts (14). On magnetic resonance imaging a further study showed that the tumor grew along the sheath, revealing a low signal intensity on T1-weighted images, but a high signal intensity on T2-weighted images. After joint intrusion by GCTTS with bone destruction, the occurrence rate of degenerative arthritis is $45 \%$ (11).

During treatment, elimination of the tumor lesions, including the soft tissue and bone, is favored. Recurrence is associated with the use of a surgical resection. In the present study, the previous tumor resection was performed at the expense of PIP function and appearance. Taking into account the patient's age, amputation was not an acceptable treatment. Complete resection of the tumor is critical to curing the disease. Due to the invasive properties of the tumor, joint stability must be considered if the joint is to be retained. The tumor cannot be completely resected, and thus the recurrence rate is high. The use of an artificial joint as a treatment for PVNS can achieve complete tumor resection, good functional recovery after surgery and reduce the likelihood of relapse (15).

Cases of GCTTS on the hand that infringes the joints, the tumor requires complete excision including the affected articular surface and joint capsule in order to have a lower rate of recurrence. The function of joints following reconstruction is a problem after the surgery. Artificial joint replacement may excise the tumor completely and also reconstruct the function of the finger joint and it is therefore a good choice for the clinic.

Artificial joint replacement surgery of the GCTTS that intruded into the distal region of the proximal phalange was successfully performed in the present case. Lesions were removed and PIP function was reconstructed.

\section{References}

1. Scott SJ and Jenkinson MD: Giant-cell tumour of the tendon sheath. J Bone Joint Surg Br 82: 1206, 2000.

2. Garg B and Kotwal PP: Giant cell tumour of the tendon sheath of the hand. J Orthop Surg (Hong Kong) 19: 218-220, 2011.

3. Athanasou NA, Quinn J, Ferguson DJ and McGee JO: Bone resorption by macrophage polykaryons of giant cell tumour of tendon sheath. Br J Cancer 63: 527-533, 1991.

4. Relwani J, Factor D, Khan F and Dutta A: Giant cell tumour of the patellar tendon sheath - an unusual cause of anterior knee pain: A case report. Knee 10: 145-148, 2003.

5. Kitagawa $\mathrm{Y}$, Ito $\mathrm{H}$, Yokoyama M, Sawaizumi $\mathrm{T}$ and Maeda $\mathrm{S}$ : The effect of cellular proliferative activity on recurrence and local tumour extent of localized giant cell tumour of tendon sheath. J Hand Surg Br 29: 604-607, 2004.

6. Demouy EH, Kaneko K, Bear HM and Rodriguez RP: Giant cell tumor of the plantar tendon sheath: Role of MR imaging in diagnosis. Case report. Clin Imaging 17: 153-155, 1993.

7. Kanagawa H, Niki Y, Matsumoto H, Kosaki N, Enomoto H, Morioka H, Toyama Y and Suda Y: Localized pigmented villonodular synovitis presenting as a loose body following minor trauma in the knee: A case report. Knee 14: 395-397, 2007.

8. Cheng XG, You YH, Liu W, Zhao T and Qu H: MRI features of pigmented villonodular synovitis (PVNS). Clinical rheumatology 23: 31-34, 2004.

9. Moore JR, Weiland AJ and Curtis RM: Localized nodular tenosynovitis: Experience with 115 cases. J hand surg Am 9: 412-417, 1984.

10. Fyfe IS and MacFarlane AU: Pigmented villonodular synovitis of the hand. Hand 12: 179-188, 1980.

11. Jones FE, Soule EH and Conventry MB: Fibrous xanthoma of synovium (giant cell tumor of tendon sheath, pigmented nodular synovitis). A study of one hundred and eighteen cases. J Bone Joint Surg Am 51: 76-86, 1969

12. Pan YW, Tian GL, Rong GW, Li C, Wang ZZ and Tian W: Giant cell tumor of tendon sheath in hand combined with bone invasion. Zhonghua Shou Wai Ke Za Zhi 20: 152-154, 2004 (In Chinese).

13. Uriburu IJ and Levy VD: Intraosseous growth of giant cell tumors of the tendon sheath (localized nodular tenosynovitis) of the digits: Report of 15 cases. J hand surg Am 23: 732-736, 1998.

14. Midletton WD, Patel V, Teefey SA and Boyer MI: Giant cell tumor of the tendon sheath: Analysis of scanographic finding. AJR Am J Roentgenol 183: 337-339, 2004.

15. Lang L and Guo W: Treatment of diffused giant cell tumors of tendon sheath by rotation hinge prosthesis. Zhongguo $\mathrm{Gu} \mathrm{Yu}$ Yuan Jie Za Zhi 5: 83-86, 2006 (In Chinese). 